\title{
Shakedown in frictional contact problems for the continuum
}

\author{
James R. Barber ${ }^{\mathrm{a}, *}$, Anders Klarbring ${ }^{\mathrm{b}}$, Michele Ciavarella ${ }^{\mathrm{c}}$ \\ a Department of Mechanical Engineering, University of Michigan, Ann Arbor, MI 48109-2125, USA \\ ${ }^{\mathrm{b}}$ Department of Mechanical Engineering, Linköping University, 58183 Linköping, Sweden \\ ${ }^{\mathrm{c}}$ CEMEC-PoliBA - Centre of Excellence in Computational Mechanics, V.le Japigia 182, Politecnico di Bari, 70125 Bari, Italy
}

Available online 4 January 2008

\begin{abstract}
Elastic systems with frictional interfaces subjected to periodic loading are often found to 'shake down' in the sense that frictional slip ceases after the first few loading cycles. The similarities in behaviour between such systems and monolithic bodies with elasticplastic constitutive behaviour have prompted various authors to speculate that Melan's theorem might apply to them-i.e. that the existence of a state of residual stress sufficient to prevent further slip is a sufficient condition for the system to shake down.

In this article, we prove this result for 'complete' contact problems in the continuum formulation for systems with no coupling between relative tangential displacements at the interface and the corresponding normal contact tractions. This condition is satisfied for the contact of two half spaces, or of a rigid body with a half space if Dundurs' constant $\beta=0$. It is also satisfied for the contact of two symmetric bodies of similar materials at the plane of symmetry. To cite this article: J.R. Barber et al., C. R. Mecanique 336 (2008).

๑) 2007 Académie des sciences. Published by Elsevier Masson SAS. All rights reserved.
\end{abstract}

\section{Résumé}

Adaptation dans les problèmes de contact avec frottement de milieux continus. Les systèmes élastiques comportant des interfaces en contact frottant, soumis à des chargements périodiques, «s'adaptent» souvent dans le sens où le glissement cesse après les premiers cycles de chargement. Les similitudes entre le comportement de tels systèmes et celui de corps monolithiques à comportement constitutif élasto-plastique ont incité divers auteurs à penser que le théorème de Melan pourrait s'y appliquer-ce qui signifierait que l'existence d'un état d'efforts résiduels suffisants pour empêcher la poursuite du glissement est une condition suffisante pour que le système s'adapte. Dans cet article, nous prouvons ce résultat pour des problèmes «complets» de contact entre milieux continus, dans le cas de systèmes sans couplage entre les déplacements tangentiels relatifs à l'interface et les tractions normales de contact correspondantes. Cette condition est satisfaite pour le contact de deux demi-espaces, ou d'un corps rigide et d'un demi-espace, si la constante $\beta$ de Dundurs est nulle. Elle est également satisfaite pour le contact de deux corps symétriques constitués de matériaux semblables. Pour citer cet article : J.R. Barber et al., C. R. Mecanique 336 (2008).

๑ 2007 Académie des sciences. Published by Elsevier Masson SAS. All rights reserved.

Keywords: Continuum mechanics; Contact problems; Fretting fatigue; Shakedown; Melan's theorem; Coulomb friction

Mots-clés : Milieux continus; Problèmes de contact ; Fatigue de fretting; Adaptation; Théorème de Melan ; Frottement de Coulomb

\footnotetext{
* Corresponding author.

E-mail addresses: jbarber@umich.edu (J.R. Barber), andkl@ikp.liu.se (A. Klarbring), mciava@poliba.it (M. Ciavarella).
} 


\section{Version française abrégée}

Dans un article précédent [6] nous avons établi une version du théorème de Melan pour le frottement de Coulomb et pour les systèmes discrets «désaccouplés », dans lesquels les déplacements relatifs tangentiels à l'interface (glissement) ne produisent pas de tractions normales de contact et le secteur de contact reste constant. Ici nous prouvons le théorème correspondant pour les milieux continus.

Nous commençons, dans l'Éq. (4), par définir l'opérateur linéaire $\mathcal{L}_{1}$ reliant la traction tangentielle $\boldsymbol{q}$ au point $(x, y)$ au déplacement tangentiel $s$ en $(\xi, \eta)$. Une condition nécessaire pour l'adaptation est l'existence d'une distribution de glissement $\tilde{\boldsymbol{s}}$ et d'une traction correspondante $\tilde{\boldsymbol{q}}$ qui, superposée à la solution ne comportant aucun glissement, engendre une traction sur tout l'interface de contact située en dessous du cône de frottement de Coulomb à tout moment pendant le cycle de chargement. Nous définissons ensuite, dans l'Éq. (11), une norme $N$ qui peut être considérée comme une mesure de la différence entre l'état instantané de glissement $\boldsymbol{s}$ et l'état $\tilde{\boldsymbol{q}}$ d'adaptation. Nous démontrons alors que la loi de frottement de Coulomb définie dans les Éqs. (7)-(9) implique que la dérivée temporelle $\dot{N}$ de cette norme est négative si le glissement se produit et nulle sinon, de sorte que le glissement provoque une évolution monotone du système vers l'état adapté. Ceci conclut la preuve du théorème.

Cette preuve repose sur l'existence et l'unicité de l'opérateur linéaire $\mathcal{L}_{1}$, qui est établie pour le cas particulier d'un corps rigide en contact avec un demi-espace élastique par réduction à un problème mixte de valeur limite, en utilisant la fonction de Green appropriée $\boldsymbol{G}$. La modification de la fonction de Green pour les corps finis comprend l'addition d'une limite liée qui n'affecte pas la validité de cet argument. En conclusion, nous montrons comment des contre-exemples au théorème de Melan pour le contact avec frottement peuvent être trouvés pour les systèmes dans lesquels les déplacements de glissement sur l'interface produisent des tractions normales de contact.

\section{Introduction}

If one or more elastic bodies with frictional contact interfaces are subjected to time-varying loads, some slip may occur at the interfaces and the instantaneous state will then generally depend on the history of loading [1-3]. A case of considerable technological importance is that in which the system is subjected to a mean load and a superposed oscillatory load, typically due to mechanical vibrations. In this case it is sometimes predicted that the system 'shakes down' in the sense that microslip occurs at the interface only during the first few cycles, after which 'stick' conditions apply throughout the contact area for all subsequent times. In effect, the accumulated microslip during the initial cycles creates a favourable state of prestress which inhibits slip in subsequent cycles. This has caused tribologists to speculate as to whether it might be possible to prove a frictional equivalent of the well-known theorem due to Melan regarding the conditions for shakedown of an elastic-plastic body subjected to oscillatory loading [4]. In fact, Churchman et al. [5] make the unequivocal statement "The Melan theorem for plasticity has a frictional equivalent."

Such a theorem might be enunciated as "If a set of time-independent tangential slip displacements at the interface can be identified such that the corresponding residual stresses when superposed on the time-varying stresses due to the applied loads cause the interface tractions to satisfy the conditions for frictional stick throughout the contact area at all times, then the system will eventually shake down to a state involving no slip, though not necessarily to the state so identified."

The present authors have recently investigated this question in the context of the discrete formulation [6]. One general result obtained was that Melan's theorem applies in two or three dimensions provided that the contact area does not change during the oscillatory loading phase and there is no coupling between normal and tangential loading. However, the theorem generally fails when such coupling exists. In a heuristic sense one would expect these results to apply equally to the continuum formulation of the problem, but the transition is by no means routine. In the present article, we shall therefore establish an equivalent theorem for continuum problems.

As in the discrete analysis [6], we restrict attention to problems in which the contact area remains constant during the oscillatory phase of the loading cycle. If this condition is not satisfied, there will be periods during which the contact area is decreasing and slip will almost always occur at the edge of the contact area under these conditions, thus preventing shakedown. For example, in the oscillatory frictional Hertzian contact problem analyzed by Mindlin and Deresiewicz [7], slip always occurs when the contact area is decreasing, unless the unloading curve in external force space is an exact reversal of the loading curve. Similar results are to be expected in any contact problem in which the contacting bodies are smooth and hence the contact area depends on the applied loads. 
Subject to this restriction, we shall show that the necessary and sufficient condition for the frictional Melan's theorem to apply is that there be no coupling between normal and tangential loading at the contact interface. In Section 2 we prove that this is a sufficient condition for the special case of indentation of an elastic half space by a rigid punch. We then extend this proof to more general contact problems in Section 3 and discuss the practical situations under which it applies. Finally, in Section 4, we establish that the absence of normal-tangential coupling is a necessary condition for the theorem by showing how counter-examples can be constructed for coupled systems.

\section{Indentation by a rigid punch}

Numerous classical contact problems have been idealized as involving two elastic half spaces or a single elastic half space indented by a rigid punch [8]. In fact, the problem of two contacting half spaces can itself be reduced to an equivalent problem in which the a single deformable half space with modified elastic properties is indented by a rigid punch. For the latter problem, there will be no normal-tangential coupling at the interface if the deformable material is incompressible $(v=0.5)$.

We therefore start our discussion by considering the problem of the incompressible elastic half space $z>0$ indented by a rigid punch in some region $A$ of its surface $z=0$. The half space is supported at infinity (displacement $\boldsymbol{u} \rightarrow 0$ as $R=\sqrt{x^{2}+y^{2}+z^{2}} \rightarrow \infty$ ) and Coulomb friction conditions hold at the interface. In response to external loads, the punch will generally execute a rigid-body translation $\boldsymbol{V}$ and rotation $\boldsymbol{\theta}$ which we represent as a six-vector $\boldsymbol{W}=$ $\left\{V_{x}, V_{y}, V_{z}, \theta_{x}, \theta_{y}, \theta_{z}\right\}$. This will result in displacements $\boldsymbol{U}(x, y)$ at the interface, given by

$$
\boldsymbol{U}(x, y)=\boldsymbol{V}+\boldsymbol{\theta} \times \boldsymbol{x}
$$

where $\boldsymbol{x}$ is a position vector.

We can then define the slip displacement $s(x, y)$ as the displacement of the surface of the half space relative to that of the punch-i.e.

$$
\boldsymbol{s}(x, y)=\boldsymbol{u}(x, y, 0)-\boldsymbol{U}(x, y)
$$

Since it is assumed that contact is maintained between the punch and the half space throughout $A$ at all times, it follows that the vector $s$ is confined to the $x y$-plane.

Following Klarbring et al. [6], we define the traction $\boldsymbol{r}(x, y)$ exerted by the punch on the surface of the half space as

$$
\boldsymbol{r}(x, y)=\boldsymbol{r}^{w}(x, y)+\boldsymbol{q}(x, y)
$$

where $\boldsymbol{r}^{w}$ is the traction that would be generated by the external forces if the punch were welded to the half space such that all slip were prevented $(s(x, y)=\mathbf{0},(x, y) \in A)$. It follows that $\boldsymbol{q}$ represents the modification to the tractions due to the slip displacement $s$ and this dependence can be recognized by the formal statement

$$
\boldsymbol{q}(x, y)=\mathcal{L}_{1}\{\boldsymbol{s}(\xi, \eta)\}
$$

where $(x, y),(\xi, \eta) \in A$ and $\mathcal{L}_{1}$ is a linear operator. We shall discuss the properties of $\mathcal{L}_{1}$ in Section 2.3 below. Here it suffices to note that the uncoupling condition implies that $\mathcal{L}_{1}\{s\}$ and hence $q$ is confined to the $x y$-plane for all $s$.

\subsection{The Coulomb friction law}

The normal contact pressure $p(x, y)>0$ can be defined as

$$
p(x, y)=\boldsymbol{k} \cdot \boldsymbol{r}(x, y)
$$

where $\boldsymbol{k}$ is the unit vector in the $z$-direction, and is therefore also the inward normal to the surface of the half space. We also define the tangential (frictional) traction vector $t$ as

$$
\boldsymbol{t}=\boldsymbol{r}-p \boldsymbol{k}
$$

The Coulomb friction law can then be stated as 


$$
\begin{aligned}
& |\boldsymbol{t}| \leqslant f p \\
& |\boldsymbol{t}|<f p \quad \Longrightarrow \quad \dot{\boldsymbol{s}}=\mathbf{0} \\
& 0<|\boldsymbol{t}|=f p \quad \Longrightarrow \quad \dot{\boldsymbol{s}}=-\lambda \boldsymbol{t}, \lambda \geqslant 0
\end{aligned}
$$

where $f$ is the coefficient of friction and a superposed dot denotes the time derivative. Condition (7) defines the classical Coulomb criterion for stick-i.e. that the ratio of the magnitude of the tangential traction to the normal compressive traction be no greater than the coefficient of friction. This restricts the admissible set of traction vectors $\boldsymbol{r}$ to a conical region known as the Coulomb friction cone. Condition (8) states that as long as $\boldsymbol{r}$ is strictly in the interior of this cone, there can be no slip. Condition (9) is the frictional analogue of the flow rule in plasticity and states that during slip, the frictional traction $\boldsymbol{t}$ must oppose the direction of the local slip velocity $\dot{\boldsymbol{s}}$.

\subsection{A shakedown theorem}

A necessary condition for the system to shake down is that there exists some time-independent distribution of slip displacement $\tilde{\boldsymbol{s}}(x, y)$ such that

$$
\tilde{\boldsymbol{r}}=\boldsymbol{r}^{w}(x, y)+\tilde{\boldsymbol{q}}
$$

lies within the Coulomb friction cone for all $x, y$ and for all times $t$, where $\tilde{\boldsymbol{q}}=\mathcal{L}_{1}\{\tilde{\boldsymbol{s}}(\xi, \eta)\}$. We shall also refer to $\tilde{\boldsymbol{s}}(x, y)$ as a safe distribution of slip displacement if the strict inequality is always enforced in (7). Physically, a safe distribution of slip displacement is one that ensures that no point in the contact area reaches the limit state of incipient slip during the steady-state loading cycle. As in the analogous plasticity problem, $\tilde{\boldsymbol{s}}$ is not generally unique. ${ }^{1}$

We next define the norm

$$
N=\frac{1}{2} \int_{A}(\boldsymbol{s}-\tilde{\boldsymbol{s}}) \cdot(\boldsymbol{q}-\tilde{\boldsymbol{q}}) \mathrm{d} A
$$

which is a measure of the difference between the instantaneous slip displacement $\boldsymbol{s}$ and the shakedown slip displacement $\tilde{\boldsymbol{s}}$. We shall consider the system to shake down if the time derivative $\dot{N}<0$ whenever $\dot{\boldsymbol{s}} \neq \mathbf{0}$. In other words, any slip that occurs causes the system to approach $\tilde{\boldsymbol{s}}$ in the sense of the norm $N$. Clearly $\dot{N}=0$ when $\dot{\boldsymbol{s}}=\mathbf{0}$ and hence $N$ is then a non-increasing function of time. This condition recognizes that the final shakedown state of the system might differ from $\tilde{\boldsymbol{s}}$, giving $N>0$ and $\dot{N}=0$. It also places no restriction on the number of load cycles or the time required to achieve shakedown, so the possibility of monotonic asymptotic approach to a zero or non-zero value of $N$ is also open.

Theorem. If there exists a safe time-independent shakedown slip displacement vector $\tilde{\boldsymbol{s}}$, the actual slip displacement $\boldsymbol{s}$ will approach $\tilde{\boldsymbol{s}}$ in the sense that $\dot{N}<0$ whenever $\dot{\boldsymbol{s}} \neq \mathbf{0}$ at any point $(x, y) \in A$.

Proof. Differentiating (11) with respect to time, we have

$$
\dot{N}=\frac{1}{2} \int_{A} \dot{\boldsymbol{s}} \cdot(\boldsymbol{q}-\tilde{\boldsymbol{q}}) \mathrm{d} A+\frac{1}{2} \int_{A}(\boldsymbol{s}-\tilde{\boldsymbol{s}}) \cdot \dot{\boldsymbol{q}} \mathrm{d} A
$$

since $\tilde{\boldsymbol{s}}, \tilde{\boldsymbol{q}}$ are time-independent.

Betti's reciprocal theorem applied to this elastic system shows that

$$
\int_{A} s_{1} \cdot \boldsymbol{q}_{2} \mathrm{~d} A=\int_{A} s_{2} \cdot \boldsymbol{q}_{1} \mathrm{~d} A
$$

\footnotetext{
1 In the analogous two dimensional discrete case, the problem of determining a suitable distribution $\tilde{\boldsymbol{s}}$ is one of the feasibility of a system of linear constraints which is well known in mathematical programming. If we add the further objective of obtaining the largest external force such that a shakedown vector $\tilde{\boldsymbol{s}}$ exists, we obtain a Linear Programming (LP) problem. Such a problem was formulated and solved for several applications by Björkman and Klarbring [9].
} 


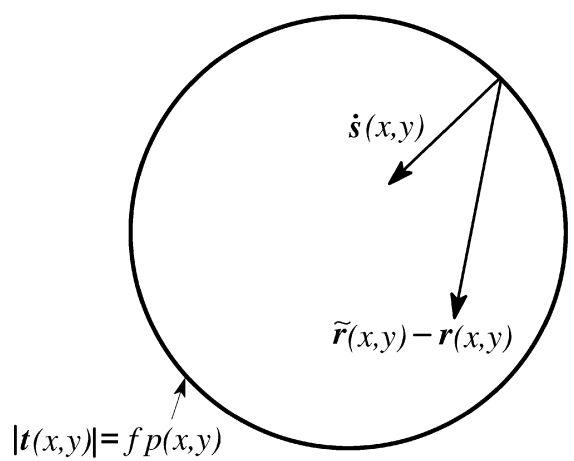

Fig. 1. The vector $(\tilde{\boldsymbol{r}}(x, y)-\boldsymbol{r}(x, y))$ must be directed into the circle from the point $\boldsymbol{r}(x, y)$ on the boundary.

where $\boldsymbol{q}_{i}=\mathcal{L}_{1}\left\{\boldsymbol{s}_{i}\right\}, i=1$, 2. It follows that the two integrals in (12) are equal, since $\dot{\boldsymbol{q}}=\mathcal{L}_{1}\{\dot{\boldsymbol{s}}\}$ from timedifferentiation of (4). We can therefore write

$$
\dot{N}=\int_{A} \dot{\boldsymbol{s}} \cdot(\boldsymbol{q}-\tilde{\boldsymbol{q}}) \mathrm{d} A=\int_{A} \dot{\boldsymbol{s}} \cdot(\boldsymbol{r}-\tilde{\boldsymbol{r}}) \mathrm{d} A
$$

where the second expression is obtained by substituting for $\boldsymbol{q}, \tilde{\boldsymbol{q}}$ from (3), (10) respectively.

If the point $(x, y)$ is slipping $(\dot{\boldsymbol{s}}(x, y) \neq \mathbf{0})$, the traction $\boldsymbol{r}(x, y)$ must lie on the friction cone and $\tilde{\boldsymbol{r}}(x, y)$ must lie strictly within the same cone. Furthermore, since $\boldsymbol{q}$ is confined to the $x y$-plane, we have

$$
\boldsymbol{r} \cdot \boldsymbol{k}=\tilde{\boldsymbol{r}} \cdot \boldsymbol{k}=\boldsymbol{r}^{w} \cdot \boldsymbol{k}=p(x, y)
$$

from (3), (10), (5). Eq. (14) states that both $\boldsymbol{r}$ and $\tilde{\boldsymbol{r}}$ must lie in a plane perpendicular to the $z$-direction whose intersection with the friction cone is the circle of radius $f p(x, y)$ in Fig. 1. The Coulomb friction flow rule (9) demands that $\dot{\boldsymbol{s}}(x, y)$ be in the direction pointing towards the centre of the circle from the point on the circumference corresponding to $\boldsymbol{r}(x, y)$, as shown in the figure.

It is clear from Fig. 1 that this requires that $\dot{\boldsymbol{s}} \cdot(\tilde{\boldsymbol{r}}-\boldsymbol{r})>0$ and hence that the integrand of (13) is negative or zero for all $(x, y)$. It follows that $\dot{N}<0$ whenever there is slip at any point in the contact area $A$, thus completing the proof.

\subsection{The operator $\mathcal{L}_{1}$}

The above theorem depends on the existence of an operator $\mathcal{L}_{1}$ such that

$$
\boldsymbol{r}(x, y)=\boldsymbol{r}^{w}(x, y)+\mathcal{L}_{1}\{s(\xi, \eta)\}
$$

In this section, we shall consider the problem of determining such an operator.

We first note that the surface displacements of the elastic half space $z>0$ due to a point force $\boldsymbol{F}$ applied at the origin can be written [10]

$$
\boldsymbol{u}(x, y, 0)=\boldsymbol{H}(x, y) \boldsymbol{F}
$$

where

$$
\boldsymbol{H}(x, y)=\frac{1}{2 \pi \mu r}\left[\begin{array}{ccc}
(1-v)+\frac{v x^{2}}{r^{2}} & \frac{v x y}{r^{2}} & \frac{(1-2 v) x}{2 r} \\
\frac{v x y}{r^{2}} & (1-v)+\frac{v y^{2}}{r^{2}} & \frac{(1-2 v) y}{2 r} \\
\frac{(1-2 v) x}{2 r} & \frac{(1-2 v) y}{2 r} & (1-v)
\end{array}\right]
$$

where $r=\sqrt{x^{2}+y^{2}}$. In the present case, $v=0.5$ and hence $\boldsymbol{H}(x, y)$ simplifies to

$$
\boldsymbol{H}(x, y)=\frac{1}{4 \pi \mu r}\left[\begin{array}{ccc}
1+\frac{x^{2}}{r^{2}} & \frac{x y}{r^{2}} & 0 \\
\frac{x^{2}}{r^{2}} & 1+\frac{y^{2}}{r^{2}} & 0 \\
0 & 0 & 1
\end{array}\right]
$$


This result can be used as a Green's function to define the surface displacement due to a traction distribution $\boldsymbol{r}(\xi, \eta)$ by convolution. We obtain

$$
\boldsymbol{u}(x, y, 0)=\int_{A} \boldsymbol{G}(x, y, \xi, \eta) \boldsymbol{r}(\xi, \eta) \mathrm{d} \xi \mathrm{d} \eta
$$

where

$$
\boldsymbol{G}(x, y, \xi, \eta)=\boldsymbol{H}((x-\xi),(y-\eta))
$$

We shall write the linear relation (18) in the symbolic form

$$
\boldsymbol{u}(x, y, 0)=\mathcal{L}_{2}\{\boldsymbol{r}(\xi, \eta)\}
$$

Consider next the problem of inverting the relation (18)-i.e. determining the distribution of tractions $\boldsymbol{r}(\xi, \eta) \in A$ needed to produce prescribed displacements $\boldsymbol{u}(x, y, 0) \in A$. This is a well-posed mixed boundary-value problem in elasticity to which the uniqueness theorem applies. We note that square-root singularities in $\boldsymbol{r}$ should be anticipated at the boundaries of $A$, but this presents no difficulties to the inversion. Thus, an inverse operator $\mathcal{L}_{3}$ exists and is unique, such that (18) implies

$$
\boldsymbol{r}(\xi, \eta)=\mathcal{L}_{3}\{\boldsymbol{u}(x, y, 0)\}
$$

Substituting for $\boldsymbol{u}$ from (2), we then have

$$
\boldsymbol{r}(\xi, \eta)=\mathcal{L}_{3}\{\boldsymbol{s}(x, y)+\boldsymbol{U}(x, y)\}
$$

The traction $\boldsymbol{r}(\xi, \eta)$ acting over $A$ will have a resultant force $\boldsymbol{F}$ and moment $\boldsymbol{M}$, which we represent as a six-vector $\boldsymbol{P}=\left\{F_{x}, F_{y}, F_{z}, M_{x}, M_{y}, M_{z}\right\}$ and which can be written down in terms of the integrals

$$
\boldsymbol{F}=\int_{A} \boldsymbol{r} \mathrm{d} A ; \quad \boldsymbol{M}=\int_{A} \boldsymbol{x} \times \boldsymbol{r} \mathrm{d} A
$$

Eqs. (20) are related to the kinematic equation (1) in a certain dual sense. Inserting (1) into (19) and the result into (20) gives, on using the linearity of $\mathcal{L}_{3}$,

$$
\boldsymbol{F}=\int_{A} \mathcal{L}_{3}\{\boldsymbol{s}\} \mathrm{d} A+\int_{A} \mathcal{L}_{3}\{\boldsymbol{V}+\boldsymbol{\theta} \times \boldsymbol{x}\} \mathrm{d} A
$$

and a similar equation for $\boldsymbol{M}$. These two equations can be written in the form

$$
\boldsymbol{P}=\mathcal{L}_{4}\{s\}+\boldsymbol{K} \boldsymbol{W}
$$

where $\mathcal{L}_{4}$ is a linear operator, $\boldsymbol{K}$ is an invertible 6 by 6 stiffness matrix and $\boldsymbol{W}$ was defined in Section 2. We solve this equation to obtain $\boldsymbol{W}=\boldsymbol{W}_{1}(\boldsymbol{P})+\boldsymbol{W}_{2}(\boldsymbol{s})$. Substituting this result into (1) and the result into (19) gives Eq. (15), hence defining the operator $\mathcal{L}_{1}$.

In the uncoupled case, when (17) holds, we note that the second term in (15) will be confined to the $x y$-plane.

\section{Extension to other systems}

The discussion so far has been restricted to the case of an elastic half space indented by a rigid punch, but extension to other systems with a single plane contact interface is routine provided: (i) there is no coupling between slip displacements $s(x, y)$ and normal contact tractions; (ii) the boundary-value problem associated with determining the operator $\mathcal{L}_{1}$ (4) is well posed; and (iii) the contact is complete, so that the contact area $A$ does not change during the loading cycle. 


\subsection{Symmetric systems}

If there are two symmetric and similar deformable bodies, a frame of reference can always be chosen such that the adhered regions of the interface (where $\boldsymbol{s}=\mathbf{0}$ ) have zero tangential displacement. The displacements in the boundaryvalue problem (4) are then antisymmetric with respect to the interfacial plane and the problem can be reduced to that of a single deformable body with a reduced elastic modulus (equal to $\mu / 2$ ) in contact with a rigid punch.

If the bodies are of finite extent, the only change is that the Green's function $\boldsymbol{H}(x, y)$ (16) will take a more complicated form. However, changes in the location of the remote boundaries can only introduce bounded terms into the expression and these will not affect the invertibility of the operator $\mathcal{L}_{2}$.

\subsection{Receding contact problems}

The condition that the contact must be complete deserves some comment, since in most cases this requires that the contact area $A$ be determined from the geometry of the system-i.e. that the contacting bodies have sharp edges.

For smooth contacting bodies, one would normally expect the contact area to be a continuous function of the loads. However, Dundurs [11] showed that if the effect of load is to reduce the contact area, this happens discontinuously and the contact area then remains constant under further increase of load. He characterized such contact problems as receding, defined such that the contact area under load is equal to or smaller than that before the loads are applied. Notice that if the two contacting bodies are conforming in the sense that they can be placed in contact with zero gap before the loads are applied, the resulting contact problem is necessarily receding, since the contact area under load cannot exceed that in the unloaded condition.

A typical example comprises two identical elastic plates contacting throughout a common interface and loaded on the exposed surfaces. In such cases separation generally occurs remote from the loaded region leaving a reduced contact area which depends on the distribution, but not on the magnitude of the loads. The contact area will then be independent of time provided the external loads $f(x, y, z, t)$ are proportional at all times so that they can be written in the separated variable form

$$
\boldsymbol{f}(x, y, z, t)=\boldsymbol{f}_{1}(x, y, z) f_{2}(t)
$$

Similar arguments can be used to show that the extent of the slip zones in receding contact problems remains constant during monotonic loading [12].

For the case of two symmetrical bodies of similar materials (which also guarantees that the system is uncoupled), the external loading can be decomposed into components $f^{S}, f^{A}$ that are respectively symmetrical and antisymmetrical with respect to the interfacial plane, in which case symmetry considerations show that the normal contact tractions and the extent of the contact area are determined only by the symmetric loads $f^{S}$. Thus, the contact area will remain constant provided $f^{S}$ has the form (21) and the antisymmetric load $f^{A}$, which alone influences the frictional tractions at the interface, can vary arbitrarily in time.

\section{Counter examples}

In [6], we established the discrete form of the present theorem, but we were also able to demonstrate that, except in a few special cases with only a few degrees of freedom, counter examples disproving Melan's theorem can be found for any discrete elastic system in which coupling exists between slip displacements and normal contact tractions. A counter example for a given frictional system corresponds to any periodic loading scenario for which a safe shakedown vector $\tilde{\boldsymbol{s}}$ exists, but for which the actual system when solved incrementally does not shake down.

Specific finite element examples illustrating this behaviour were given by Björkman and Klarbring [9]. By linear programming methods, they determined the maximum periodic loading amplitude for which a safe shakedown vector $\tilde{\boldsymbol{s}}$ could be established. They then solved the same problem with a quasi-static incremental algorithm and hence established an upper bound to the amplitude at which shakedown actually occurs. In all cases, this upper bound was significantly lower than the limit established by linear programming, showing that there is a range of loading cycles where the existence of $\tilde{\boldsymbol{s}}$ is not sufficient to ensure shakedown.

The discrete arguments from [6] could be regarded as giving a convincing disproof of Melan's theorem for coupled systems, provided we accept the premise that finite element results approximate the true behaviour of the continuum 
under sufficient mesh refinement. However, specific counter examples avoiding this assumption can also be developed as in the present paper by replacing stiffness matrix operations by appropriate convolution integrals. For example, Comninou and Barber [13] considered the problem of a layer of thickness $a$ pressed against a half space of the same material by a uniform pressure $p_{0}$ and then subjected to an oscillating tangential load $Q$ at $x=0$ on the free surface. Tractions due to slip displacements were represented by convolution on a distribution of glide dislocations $B_{x}(x)$ in the slip regions - a technique which has been widely used for two dimensional crack problems [14]. They tracked the extent of the slip zones throughout the cycle and hence established the maximum amplitude of the load $Q$ for which shakedown occurred. In this problem, slip does not extend beyond the region $-2 a<x<2 a$, but the resulting slip displacements cause residual tractions throughout the interface. It is now a simple matter to construct a distribution $B_{x}(x)$ in a region further away from the load $Q$ which (i) is insufficient to cause local slip and (ii) is such as to increase the normal contact pressure in the vicinity of the load $Q$. In fact, the negative of the residual tractions represented in Figs. 6 and 7 of [13] clearly meets these conditions. Any such favourable residual traction distribution furnishes a counter example, since it raises the effective shakedown limit, but there is no way that the slip necessary to form such a distribution could occur under the influence of the cyclic load $Q$.

Such counter examples can be found for almost all coupled problems. All we need to do is: (i) find the actual shakedown limit by an incremental solution; (ii) identify a region where no slip has occurred and the tractions are always strictly within the Coulomb friction cone; and then (iii) impose slip displacements in this region in such a direction as to raise the normal contact pressure in regions that just reach the boundary of the friction cone during the loading cycle.

\section{Conclusions}

We have proved that Melan's theorem applies to continuum contact problems with frictional contact, provided the contact area does not change during the loading cycle and there is no coupling between tangential slip displacements and the normal contact tractions. In practice, this restricts the theorem to symmetric systems contacting on the symmetry plane or to the indentation of an incompressible elastic half space by a rigid punch. For systems with coupling between slip displacement and normal contact tractions, Melan's theorem can generally be disproved by counter example, and a strategy for developing such counter examples is outlined.

\section{References}

[1] J. Dundurs, M. Comninou, An educational elasticity problem with friction—Part I: Loading, and unloading for weak friction, ASME J. Appl. Mech. 48 (1981) 841-845.

[2] M. Comninou, J. Dundurs, An educational elasticity problem with friction—Part II: Unloading for strong friction and reloading, ASME J. Appl. Mech. 49 (1982) 47-51.

[3] J. Dundurs, M. Comninou, An educational elasticity problem with friction—Part III: General load paths, ASME J. Appl. Mech. 50 (1983) $77-84$.

[4] E. Melan, Theorie statisch unbestimmter Systeme aus ideal-plastichem Baustoff, Sitzungsber. d. Akad. d. Wiss., Wien 2A (145) (1936) 195218.

[5] C.M. Churchman, D.A. Hills, General results for complete contacts subject to oscillatory shear, J. Mech. Phys. Solids 54 (2006) $1186-1205$.

[6] A. Klarbring, M. Ciavarella, J.R. Barber, Shakedown in elastic contact problems with Coulomb friction, Int. J. Solids Structures 44 (2007) $8355-8365$.

[7] R.D. Mindlin, H. Deresiewicz, Elastic spheres in contact under varying oblique forces, ASME J. Appl. Mech. 75 (1953) 327-344.

[8] D.A. Hills, D. Nowell, A. Sackfield, Mechanics of Elastic Contacts, Butterworth Heinemann, Oxford, 1993.

[9] G. Björkman, A. Klarbring, Shakedown and residual stresses in frictional systems, in: G.M.L. Gladwell, H. Ghonem, J. Kalousek (Eds.), Contact Mechanics and Wear of Rail/Wheel Systems II: Proceedings of the 2nd International Symposium, University of Waterloo Press, 1987, pp. 27-39.

[10] H.M. Westergaard, Theory of Elasticity and Plasticity, Dover, New York, 1964.

[11] J. Dundurs, Properties of elastic bodies in contact, in: A.D. de Pater, J.J. Kalker (Eds.), The Mechanics of the Contact Between Deformable Bodies, Delft University Press, 1975, pp. 54-66.

[12] M. Ciavarella, A. Baldini, J.R. Barber, A. Strozzi, Reduced dependence on loading parameters in almost conforming contacts, Int. J. Mech. Sci. 48 (2006) 917-925.

[13] M. Comninou, J.R. Barber, Frictional slip between a layer and a substrate due to a periodic surface force, Int. J. Solids Structures 19 (1983) 533-539.

[14] D.A. Hills, P.A. Kelly, D.N. Dai, A.M. Korsunsky, Solution of Crack Problems: the Distributed Dislocation Technique, Kluwer Academic Publishers, Dordrecht, 1996. 